\title{
DIRECT MASS SPECTROMETRY-BASED APPROACHES IN METABOLOMICS
}

Clara Ibáñez, Virginia García-Cañas, Alberto Valdés and Carolina Simó*

Laboratory of Foodomics, Institute of Food Science Research (CIAL), CSIC. Nicolás Cabrera 9, 28049 Madrid, Spain

*Corresponding author: Carolina Simó. E-mail: c.simo@csic.es

\begin{abstract}
Metabolomics is one of the newest omics technologies concerned with the identification and quantification of small molecules in a high-throughput manner. Considering the number of different types of metabolites present in a wide dynamic range of concentrations in any single living system, still actual analytical technologies can only capture a part of the metabolome. Currently MS-based approaches yield a higher sensitivity than NMR when analyzing minimal amounts of complex mixtures. Most MS-based approaches in metabolomics involve a physical/chemical purification/fractionation prior to MS analysis, to avoid sample matrix effects, at the expenses of low high-throughput performance. In the quest to achieve the maximum highthroughput production of metabolite information in the largest possible number of samples an extensive array of direct ionization or desorption/ionization techniques have been developed and combined. In the present Chapter an overview of the main desorption/ionization techniques coupled to MS applied to direct metabolite profiling or fingerprinting is presented.
\end{abstract}

Keywords: Mass spectrometry, ambient MS, MALDI, SIMS, DESI, DART, NIMS, imaging MS 


\section{TABLE OF CONTENTS}

1. INTRODUCTION

2. MATRIX-ASSISTED AND MATRIX-FREE LASER DESORPTION/IONIZATION MS

3. DIRECT INFUSION MS

4. AMBIENT IONIZATION MS

5. IMAGING MS

6. CONCLUSIONS 


\section{INTRODUCTION}

The general approach in metabolomics is the analysis of as many low-molecular weight compounds as possible in a given sample to obtain maximal biochemical information. The reality is that considering the number of different types of metabolites in a single living system (lipids, carbohydrates and many other small compounds, such as amino acids, organic acids, nucleic acids, fatty acids, phytochemicals, minerals, etc.), still actual analytical technologies can only capture a part of the metabolome. Two analytical platforms are by far the most predominantly used for metabolomic analyses: mass spectrometry (MS) and nuclear magnetic resonance (NMR) technologies. Currently MS-based approaches yield a higher sensitivity than NMR when analyzing minimal amounts of complex mixtures. In particular, the use of high and ultra-high resolution mass spectrometers greatly improves analytical performance and offers the best combination of selectivity and sensitivity. "Conventional" methods for analyzing metabolites by MS usually involve a physical/chemical purification/fractionation prior to MS analysis. Thus, to avoid sample matrix effects hyphenation of high resolution separation techniques and MS is usually carried out at the expenses of less high-throughput performance.

There is a clear need for more rapid, high-throughput MS approaches for metabolomics studies. In the quest to achieve the maximum high-throughput production of metabolite information in the largest possible number of samples an extensive array of direct ionization or desorption/ionization techniques have been developed and combined. Using these approaches, any chromatographic or electrophoretic step prior to MS detection is avoided and thus direct analysis of samples (processed or not) is carried out. Sample introduction and ionization system used prior to MS analysis will always cause discrimination of specific metabolite classes. Thus, the choice of the most adequate methodology will require careful consideration taking into account the goal of the metabolomic work. When direct MS analysis approaches are used, ionization suppression and its effect on sensitivity are not negligible since the presence of multiple chemical species and other matrix components will have considerable impact on the ionization of metabolites. Moreover the overlapping of MS signals of isobaric species will be a common drawback of direct ionization technologies. Despite of these limitations the major potential of this approach is its highthroughput character, especially when the number of samples to be analyzed is high. Thus, by using a variety array of sample introduction/desorption/ionization techniques, metabolome screening in complex samples can be obtained in a few seconds by direct MS analysis. Moreover, through direct MS analysis the chemical composition within the spatial context of biological samples is also possible. Most MSbased approaches to analyze tissues require certain sample preparation what leads to destruction of the histology structures. MS imaging (MSI) allows the capability to capture the chemical composition within the spatial context of biological tissues. In the present Chapter an overview of the main 
desorption/ionization techniques coupled to MS applied to direct metabolite profiling or fingerprinting is presented.

\section{MATRIX-ASSISTED AND MATRIX-FREE LASER DESORPTION/IONIZATION MS}

Matrix-assisted laser desorption/ionization (MALDI) development has largely focused on high molecular weight polymers and biopolymers. With the development of new generation time-of-flight (TOF) mass analyzers with remarkable improvements in mass resolution the interest in application of MALDI-MS to small molecules has been recently renewed $[1,2]$. Using MALDI, sample is spotted on a metal plate with a solid or liquid matrix, and is co-crystallizing with a highly UV-absorbing substance, which is generally a low molecular weight compound. When compared with direct infusion MS-based approaches MALDIMS has some advantages, specially its high tolerance towards salts and buffers, and in addition, the amount of sample consumed during analysis is very small. Application of MALDI-MS to small molecules typically involves a target approach [1,2], and global metabolite analysis is relatively recent. One of the main limitations of MALDI-MS in metabolite analysis is signal suppression due to matrix background ions (in the low-mass range , $\mathrm{m} / \mathrm{z}<700$ ) from conventional matrices such as 2,5-dihydroxybenzoic acid (DHB) and $\alpha$-cyano-4-hydroxycinnamic acid (CHCA)) which interferes with the analysis of the analyte. The use of a fluorophenyl porphyrin as matrix, instead of a standard low molecular weight matrix did not produce ions in the low-mass region (100-500 Da) [3]. Using this matrix, determination of the fatty acid composition in a variety of vegetable oils was carried out by MALDI-TOF MS with minimum sample treatment [4]. To improve the analysis of low-mass molecules Guo et al. [5] developed a novel approach to suppress the production of matrix-related background ions (from the common matrix CHCA) in MALDI by adding the surfactant cetrimonium bromide (CTAB). As a result of the CHCA related ion background suppression, very clean mass spectra was routinely obtained in the low-mass range. The use of small, non-polar polymers (oligomers) based on oligothiophene or oligobenzodioxin as matrices allowed the analysis of model small molecular weight compounds [6]. It was suggested that the mechanisms for forming positive ions was based on charge transfer, rather than proton transfer. In a different work the use of ionic liquid-based matrices [7] or other matrix-assisted compounds like 9aminoacridine [8,9] have been presented for high-throughput metabolomics applications. These strategies are in continuous development for a more amenable analysis of small molecules minimizing matrix interferences.

Significant effort has also been made to develop laser desorption/ionization (LDI) techniques that can be performed without matrix, allowing placing the sample directly onto a surface. Sample preparation is 
simplified in this case, and inhomogeneous co-crystallization processes of the sample and matrix are avoided. One of the first matrix-free LDI-MS method was introduced in 1999 by G. Siuzdak by the development of LDI on porous silicon (desorption ionization on porous silicon, DIOS) [10]. The porous silicon substrate is easily obtained by electrochemical anodization of crystalline silicon in hydrofluoric acid-based solutions. This strategy was presented as an encouraging matrix-free strategy to counteract these interferences. Both unoxidized [11] and oxidized [12] porous silicon surfaces showed to be a successful and simple method for high-throughput analysis of metabolites. However this approach has still some limitations, for example, due to the infiltration of analytes into the pores of the silicon, the control of the position and size of the crystalized analytes is difficult, and thus, this results in a less effective energy transfer when the analyte is not in the silicon surface. Nanostructure-initiator mass spectrometry (NIMS) was introduced as an alternative matrix-free approach to DIOS to produce low background and high-sensitivity MS measurements [13]. NIMS uses initiator molecules trapped in nanostructured surfaces or 'clathrates' to release and ionize intact molecules adsorbed on the surface. A list of compounds used as initiators for NIMS can be found elsewhere [14]. When the surface is heated with a laser (or ion beam), the initiator violently erupts from the pore, triggering the desorption-ionization of the analyte. In contrast to MALDI these compounds do not absorb UV energy, analytes do not cocrystallized with the initiator, and most of them do not ionize. NIMS-MS has been used for direct biofluid analysis (blood and urine) [13]. Nanostructure-assisted LDI (NALDI) is a patented matrix-free technology (NALDI ${ }^{\mathrm{TM}}$ chip, from Bruker Daltonics). The nano-material on the target absorbs the laser energy and allows for the desorption/ionization process of the analyte [15]. As a result of the absence of the matrix, mass spectra present very low chemical background. Disposable nano-structured target plates are commercially available, and they have been used in a variety of small molecule analysis, such as phospholipids [16]. Many other matrix-free methods have been developed for laser desorption/ionization of small molecules, with potential applications in metabolomics. A variety of surface properties have been studied for matrix-free ionization of small molecules, for example: diamond nanowires [17] sol-gel derived silver-nanoparticles-impregnated thin biofilm [18], nanoporous gallium nitride-silver nanoparticles [19], metal oxide surfaces [20], nanofilament silicon [21], etc. Comprehensive reviews of innovative technologies using energy-absorbing materials for matrix-free LDI-MS have been recently published [22-24].

\section{DIRECT INFUSION MS}


Among atmospheric pressure ionization (API) techniques, both electrospray ionization (ESI) and atmospheric pressure chemical ionization (APCI) ion sources are surely the most employed techniques in MS analysis using direct infusion approaches. When using direct infusion approaches sample typically needs to be treated to dissolve the compounds of interest in the appropriate solvent. By using direct infusion MS-based approaches sample preparation can be considered a "bottleneck". In this sense, automated multi-well devices for metabolite purification provide the highest throughput in sample preparation. When large-scale multi-batch experiments are designed, robust workflows have to be developed to minimize experiment analytical variation [25]. Lin et al. compared the classification and biomarker discovery capacities of direct infusion ESI-MS and liquid chromatography (LC)-MS [26]. For that purpose, serum samples from kidney cancer patients and healthy controls were analyzed by both analytical techniques. It was observed that direct infusion ESI-MS had comparable classification and prediction capabilities to LC-MS but consumed only $\sim 5 \%$ of the analysis time. In contrast, biomarker discovery of LC-MS (48 variables) was better than that of direct infusion ESI-MS (23 variables).

By using direct infusion approach based on ESI-MS, multi-dimensional mass spectrometry-based shotgun lipidomics (MDMS-SL) has also demonstrated to be a successful innovative approach in non-targeted analysis of lipids [27]. Using this approach a 2D mass spectrum is constructed. The first dimension is composed by the molecular ions in $\mathrm{m} / \mathrm{z}$ values, while the second dimension is comprised of the mass corresponding to the neutrally lost fragments or the monitored fragment ions in $\mathrm{m} / \mathrm{z}$ values (Fig. 1). The cross peaks of a given primary molecular ion in the first dimension with the second dimension represent the fragments of a given molecular ion. The major difficulty of this approach is the accurate interpretation of spectra. On the other hand, the main drawback of this methodology is ion suppression that can be partially avoided with exhaustive sample purification.

Direct infusion MS is a very interesting approach especially when sample characteristics allow MS analysis with minimum sample treatment. Thus, ESI can be used to directly ionize analytes in liquid samples in a high electric field. A small flow of the liquid sample is conducted through a capillary to the high electric field for ESI. Usually, sample solutions must be carefully cleaned and filtered to avoid potential capillary blocking. Following this idea direct infusion ESI-FTICR (Fourier transform ion cyclotron resonance) MS of coffee drink combined with partial least-squares multivariate statistical analysis was successfully employed to predict the blend composition of commercial coffee varieties [28]. In a different work, minimal sample manipulation was carried out to obtain detailed molecular composition of edible oils and fats analyzed by flow injection ESI-Orbitrap MS for quality assessment and authenticity control purposes [29]. However, when using direct infusion approaches sample typically needs to be treated to dissolve the compounds of interest in the appropriate solvent. 
Generally, each direct MS analysis methods discriminate differently and specifically against certain physical-chemical properties of analytes. Nordstrom et al. followed a multiple ionization MS strategy using ESI, APCI, MALDI and DIOS for increased coverage of the metabolome in biological samples [30]. From the obtained results it was concluded that for a true global metabolomics study multiple ionization technologies are required.

\section{AMBIENT IONIZATION MS}

Recently, a new family of techniques that operate under ambient conditions has emerged with the attempt to minimize the need for sample preparation and separation (purification/fractionation) prior to MS analysis by combining the sampling and ionization/desorption processes into a single step. In this sense, since the early 2000s the highest efforts are aimed at the performance of these "ambient" ionization techniques. Ambient ionization refers to a variety of combinations of sample introduction systems, and desorption and ionization methods that allow direct analysis of sample surfaces in open-air conditions with little or no sample pretreatment, and in most cases through noninvasive procedures [31-34]. For this reason, MALDI and "traditional" API techniques such as ESI and APCI are not considered to belong to this group since they usually still require extensive sample preparation and/or vacuum conditions.

The development of ambient MS was initiated with the introduction of desorption electrospray ionization (DESI) by Cooks in 2004 [35]. Since then, a variety of possibilities combining different desorption and ionization methods have been developed. Almost 30 ambient sampling/ionization approaches were involved in MS analysis in the 2-year period 2009-2010, as reviewed by Harris et al. [36]. Among them, DESI and direct analysis in real time (DART) were the two most prevalent techniques.

DESI is derived from traditional electrospray ionization, and as described by the developers, DESI shares the advantages of the matrix-free DIOS and the advantageous production of multiply charged ions of ESI. DESI-MS has demonstrated its usefulness in high-throughput differential metabolomics of biological samples with minimal sample preparation [37]. Using DESI approach electrosprayed aqueous droplets are directed at a surface of interest in air and act as projectiles desorbing ions from the surface as a result of electrostatic and pneumatic forces. As can be seen in Fig. 2, electrical charge applied to the solution produces charged droplets, which in aqueous solutions lead to an excess of hydronium ions $\left(\mathrm{H}_{3} \mathrm{O}^{+}\right)$or hydroxide ions $\left(\mathrm{OH}^{-}\right)$, and hence to protonated or deprotonated analytes, which are observable in the positive ion and negative ion modes, respectively [38]. 
DESI-MS allowed differentiation between diseased (lung cancer) and healthy mice urine samples [37]. DESI-MS has also been demonstrated to be a promising tool in food safety control. Thus, successful analysis of a group of agrochemicals (insecticides, herbicides, and fungicides) was carried out spotting fruit and vegetable extracts onto conventional smooth poly(tetrafluoroethylene) (PTFE) surface [39]. However the real potential of this approach was demonstrated by the direct DESI-MS/MS analysis of fruit peels from market samples without any further sample treatment [39]. DESI-MS also allowed the rapid analysis of sulfur volatiles in several onion varieties to distinguish phenotypes (tearless and normal) by simply scratching leaves and recording the extractable ions for $<0.5 \mathrm{~min}$ [40]. In this field, DESI-MS implemented in portable instruments is being performed in Cooks's laboratory for a rapid, in situ, direct qualitative and quantitative (ultra)trace analysis of agrochemicals in foodstuffs.

DESI was followed by DART in 2005 [41]. DART can be considered an API-related technique and is based on the thermo-desorption of condensed-phase analytes by a (distal) plasma discharge in a heated gas stream (helium or nitrogen). Metastable atoms generated from gas interact with ambient molecules, such as water, to create gas-phase ionic reagents which in turn react and ionize analyte on a surface or present as a vapor in the atmosphere (Fig. 3) [38]. DART-MS has been applied to the analysis of small molecules in numerous types of samples without prior preparation [42]. DART is capable of analyzing low to high polarity compounds (up to $1 \mathrm{kDa}$ ) in both negative-ion and positive-ion modes, however it is not really suitable for ionic compounds. Analysis of small molecules in plasma samples without sample preparation has been demonstrated by DART-MS [43]. Even living organisms can be subjected to DARTMS analysis [44]. Although more slowly than DESI-MS, DART-MS is now beginning to deliver its potential in Metabolomics. DART-MS proved to be a powerful analytical technique for rapid metabolic fingerprints of human serum [45, 46]. Metabolic fingerprints obtained by DART-MS of tomato and pepper have been recently reported for classification purposes (crops grown under organic vs. conventional conditions) [47]. Monitoring tea fermentation/maturation of tea through a non-targeted metabolite analysis approach was also possible by using DART-MS [48]. Hajslova et al. have critically reviewed DART-MS applications for food quality, safety and authentication purposes [49]. As an example of its utility in food safety, DART-MS permitted the measurement of xenobiotics in real time on the fruit peel [50]. As occurred with other novel ambient desorption ionization techniques, maximal performance is achieved when ultrahigh resolution mass spectrometers are used, as is the case of FTICR MS [51] or Orbitrap MS [52]. As an example, DART-Orbitrap MS showed its potential in the metabolic profiling of flavonoids and other phenolic compounds in propolis [53].

Paper spray ionization is another recently developed ionization method for a fast direct MS analysis of complex mixtures on a paper substrate in open environment conditions [54]. It shares characteristics of 
ESI and ambient ionization methods. Sample is loaded by dropping or by wiping the surface of interest onto a paper of triangle shape. The electrospray is induced from the sharp tip of the triangular paper wetted with a small amount of sample by applying a high voltage (about $5 \mathrm{kV}$ ). The geometry of the paper substrate, the onset voltage for spray, and the sample load have been investigated for their effects on the ionization efficiency. The capabilities of paper spray ionization have been mostly demonstrated for the direct analysis of biological samples in drug monitoring applications [55]. Thus, the measurement of therapeutic drugs and their metabolites in dried blood spots. Paper spray ionization method has also been used as a direct sampling ionization method for MS analysis of additives in foods [56]. Thus, a piece of paper wetted with methanol was used to wipe a $10 \mathrm{~cm}^{2}$ area on the peel of a lemon. Although the identification of particular compounds from food surfaces showed the potential of this approach, a further development should be carried out for its implementation in non-targeted metabolomics applications. Of particular interest was the modification based on paper spray ionization made by its own developers [57,58], in which a fresh triangular piece of onion and spinach leaves served as both sample and substrate (Fig. 4). Using this original approach, stress-induced changes in glucosinolates could be followed on the minute time scale.

\section{IMAGING MS}

The use of imaging mass spectrometry (IMS) for surface-based analysis of metabolites in tissue sections/surface is a remarkable novel approach [59], particularly in metabolomics [60]. Recent advancements in the field of IMS have specifically been reviewed and discussed showing the great potential of this technique in small molecule analysis [61, 62]. Through a computer-controlled xy stage to the ionization source, the surface of the sample is typically scanned with a local desorbing and ionizing probe, and the generated ions from the surface are analyzed by MS [63]. In Fig. 5 basics of IMS are represented [64]. Thus, 2D and 3D constructions of chemical abundance of metabolites from cells or tissues allow a deeper knowledge concerning the spatial organization of metabolic processes, cell-to-cell communication, molecular transport, etc. Since altered chemical/molecular distributions are diagnostic for diseases, a direct examination of biological processes will result in a better understanding of the pathophysiology. On the other hand, the application of IMS to drug discovery/development is particularly attractive because it provides the opportunity to detect the localization of a certain drug and its metabolites, as well as detect metabolite changes as a result of drug administration. Although the majority of the IMS experiments are based on imaging animal tissue sections or small tumor biopsies, IMS is starting to be applied to three-dimensional cell and tissue culture systems. Typically, samples from 
biological sources such as a biopsy, a post-mortem organ, tumor section, as well as plants and single cells, are the object of an imaging study. In IMS rigorous sample preparation is vital to achieve the most accurate, reproducible and validated data as possible [65]. In most cases dissection of the sample is generally followed by freezing the tissue (in liquid nitrogen or isopentane) and storing at $-80{ }^{\circ} \mathrm{C}$ until use. Different types of desorption/ionization sources are currently in use or being developed to be used in IMS [66]. Each technique has advantages over the alternatives in terms of sensitivity, selectivity, spatial resolution or sample preparation. Brief descriptions of most used desorption/ionization technologies IMS are described below.

MALDI-IMS was pioneered by R. Caprioli [67] in the late 1990s to generate ion images of peptides and proteins in biological samples. Without considering proteomics and peptidomics applications, lipids have been one of the first targets in IMS studies. In IMS spatial resolution is usually dependent on the type of ionization employed, and it refers to the minimum distance between two objects in an image at which they can be distinctly discerned. In MALDI-IMS lateral spatial resolution is limited by the laser beam diameter (shape and focusing) and the size of the matrix crystal.

Since the early works on MALDI-IMS to produce molecular images directly from tissue sections [67] a growing interest to monitor the distribution of a wide variety of compounds (metabolites, lipids, peptides, proteins and xenobiotics) has been observed in the last years. A variety of applications can be found in literature. As an example, MALDI has demonstrated its versatility in the analysis of the spatial distribution of metabolites in plant-based applications [68]. Zaima et al. optimized an IMS method using MALDI and conventional DHB matrix for nutritional food factors screening in rice [69]. The same research group also applied a similar approach for authenticity assessment of beef origin through metabolomic analysis [70]. As already discussed in the Section 2 one of the main problems of the use of MALDI in metabolomics application is the formation of matrix-related peak interferences. By using 9aminoacridine (9-AA), already mentioned in Section 2, only a few peaks derived from the matrix were observed in the low mass range $(\mathrm{m} / \mathrm{z} \sim 500)$. As an example, a sensitivity of some tens of attomoles per pixel and without any chemical labeling of 13 primary metabolites was obtained on rat brain sections using 9-AA as a matrix [71] (Fig. 6). Whole-body sections analysis from an animal is also possible for label-free tracking by MALDI-IMS of both endogenous and exogenous compounds with spatial resolution and molecular specificity [72]. MALDI-IMS using 9-AA as a matrix has also been proved to be sensitive enough for the detection of metabolites and for 2D imaging with single-cell sensitivity [73]. MALDI-MSI involving both CHCA and 9-AA was used to analyze the distribution of metabolites (amino acids, sugars, phosphorylated metabolites) in wheat seeds at different stages of development and under temperature stress [74]. 
In addition of MALDI, matrix-free LDI techniques have been proved to be useful in IMS studies of small molecules [62]. Thus, NIMS has also been proved to be a highly sensitive matrix-free method (in which functionalized surfaces are used to absorb the laser, eliminating the need for matrices) for tissue imaging in metabolomics [75, 76]. It has been described that NIMS surface can be easily treated or modified with different chemical initiators. As a result, distinct metabolite profiles from the same biological sample can be obtained. For instance, coating NIMS surface with cationization agents $\left(\mathrm{AgNO}_{3}\right)$ permitted the acquisition of images of brain sterol localization in a mouse model. Abnormal cholesterol biosynthesis in pathological brain tissues could be identified following this approach [77]. Sturm et al. compared images obtained by MS by using both NIMS and MALDI for neuropeptide and lipid imaging in a crustacean model organism (Cancer borealis) brain [78]. Similar lipid profiles were obtained using both strategies; however, MALDI-IMS gave better performance in the neuropeptide imaging experiments than NIMS. Other interesting applications have been published using other matrix-free methodologies, such as NALDI. As an example, NALDI-MS images of tumors through lipid analysis was demonstrated to be an encouraging technology for biomarker discovery [79].

MALDI-IMS is still limited to about $20 \mu \mathrm{m}$ spatial resolution (with 10 to $50 \mu \mathrm{m}$ the most commonly achievable spatial resolution at this time), typically obtained with commercial ion sources [80]. Recently Zavalin et al. demonstrated that $5 \mu \mathrm{m}$ spatial resolution can be achieved for MALDI-IMS instruments by spatial filtration of the laser beam by using a $25 \mu \mathrm{m}$ ceramic pinhole filter [81]. Secondary ion mass spectrometry (SIMS), developed in the 1960s [82], offers a complementary or alternative method to MALDI- and other matrix-free LDI-MS methods for the acquisition of higher spatial resolution images. In SIMS, a focused high energy primary ion beam (such as $\mathrm{Cs}^{+}, \mathrm{Au}^{3+}, \mathrm{Bi}_{5}{ }^{+}$or $\mathrm{C}_{60}{ }^{+}$) is used to directly bombard at the sample surface. The primary ions transfer their energy to molecules on the surface resulting in the desorption of ionized molecules (secondary ions) which are then analyzed by MS (Fig. 7). Secondary ions are either positive or negative, depending on the primary ions identity. Due to the use of primary ion beam images can be acquired at high lateral resolution ( $\geq 50 \mathrm{~nm}$ ) [83]. In contrast to MALDI, SIMS produces higher fragmentation of desorbed ions what makes desorption of large intact molecules very difficult. Due to high fragmentation rate and low ionization efficiency, the size of biological molecules detected by SIMS analysis is limited $(\sim 2 \mathrm{kDa})$. The different types of ion beams can be used to increase the intact ion yield of larger or more labile compounds. As pointed by Fletcher et al. sensitive SIMS-IMS still remains the requirement to significantly increase the secondary ion yields [84]. The use of SIMS in lipid MS imaging has been recently evaluated, and successfully applied to elucidating a number of biological processes [85]. SIMS offers particularly powerful capabilities in single cell MS imaging area [86]. TOF MS are most commonly coupled with SIMS and MALDI sources for surface imaging. But the 
ultra-high mass resolving power capabilities of FTICR MS can provide better mass identification capabilities of secondary ions with high specificity in tissue imaging [87]. However, analysis time continues to be lengthy, and lower spatial resolution capabilities were obtained when compared to SIMSTOF IMS platforms.

In addition to laser beam- or ion beam-based methods, other matrix-free-based methods using a gas/liquid jetstream can also be used for desorption and ionization of compounds from the sample surface. This is the case of DESI method under ambient conditions. DESI has very recently started to be explored for the analysis of small compounds imaging on intact surfaces [88]. In contrast to MALDI and SIMS in which the sample must be confined (in most cases) in a high-vacuum region of the instrument, in DESI-IMS the tissue surface is maintained at atmospheric pressure in open-air and probed with a focused spray of charged microdroplets of a polar solvent. Lateral spatial resolution provided by DESI is typically $250 \mu \mathrm{m}$ [89], lower than MALDI or SIMS. In contrast, DESI usually requires less sample preparation. DESI-MSI has become an attractive tool for discovering the distribution of secondary metabolites in plants [90] and lipids in variety of tissue samples [91]. Recently Li et al. [92] explored the capabilities of IMS using DESI for the study of secondary metabolites in barley leaf surface. Although direct DESI analysis of the untreated leaves was not possible, it was certainly possible by stripping and analyzing the epidermis from the leaf. Thus, a number of hydroxynitrile glucosides from three different cultivars of barley were successfully identified and imaged throughout the leaves surfaces. DESI-IMS has been recently applied for a better understanding of the molecular signatures of plant surfaces thin layer chromatographic imprints of leaves/petals of several plants [93]. In Fig. 8 a particular example of the images of selected ions from petals and a leaved TLC imprints, is shown.

Laser ablation electrospray ionization (LAESI) is especially designed for biological samples containing water. Using this technique, a focused mid-IR laser excites the $\mathrm{OH}$ vibrations in a sample's water molecules. Phase explosion causes a rapid microscale ablation, ejecting a mixture of molecules, clusters, and particulate matter from the sample surface. Variations in the water content or tensile strength of tissues can affect the spatial resolution. LAESI was successfully applied to 3D imaging MS of metabolites in leaf tissues, obtaining specific secondary metabolite accumulation patterns that correlate with the biochemical roles of these chemical species in plant defense and photosynthesis [94]. The feasibility of metabolite imaging using LAESI on chemically untreated sections of brain tissue at atmospheric pressure has also been demonstrated [95]. Among all mentioned methodologies only DESI and LAESI techniques operate exclusively under atmospheric pressure, and sample treatment is minimum, which makes them suitable approaches for screening purposes (analysis of large sample sets). Both DESI and LAESI are comparable in terms of lateral spatial resolution (300-400 $\mu \mathrm{m})$ [96], and thus, 
further improvements are needed in this matter. Other less common ambient desorption/ionization techniques allow IMS to be performed under atmospheric pressure on untreated samples outside the MS. Latest developments and applications in this field have been reviewed by Wu et al. [64].

\section{CONCLUSIONS}

A variety of novel direct MS-based approaches with promising utility in metabolomics has been introduced in this chapter. Among them, ambient MS is a very active area of research to perform highthroughput analysis, and it is expected that ambient MS will become routine biochemical tools for metabolomics applications. On the other hand, IMS is a rapid growing technology, and although it is still in an early stage in metabolomics field, the potential of metabolite imaging in tissue sections is enormous. IMS offers complementary information to conventional metabolomics. However, the simultaneous and spatially resolved detection of a broad range of metabolites with high sensitivity is still a challenging issue in IMS. Imaging acquisition speed, resolution and data mining tools need to be further developed. As the IMS field grows new instrumentation and methods, such as improved lasers, matrix/solvent combinations, and advanced imaging software will maximize sensitivity and identification capabilities. 


\section{REFERENCES}

[1] L.H. Cohen and A.I. Gusev, Anal. Bioanal. Chem., 373: 571-586, 2002

[2] J.J. van Kampen, P.C. Burgers, R. de Groot, R.A. Gruters and T.M. Luider, Mass Spectrom. Rev., 30: 101-120, 2011.

[3] F.O. Ayorinde, P. Hambright, T.N. Porter and Q.L. Keith Jr., Rapid Commun. Mass Spectrom., 13: 2474-2479, 1999

[4] F.O. Ayorinde, K. Garvin and K. Saeed, Rapid Commun. Mass Spectrom., 14: 608-615, 2000

[5] Z. Guo, Q. Zhang, H. Zou, B. Guo and J. Ni, Anal. Chem., 74: 1637-1641, 2002

[6] A. Woldegiorgis, F. von Kieseritzky, E. Dahlstedt, J. Hellberg, T. Brinck and J. Roeraade, Rapid Commun. Mass Spectrom., 18: 841-852, 2004

[7] S. Vaidyanathan, S. Gaskell and R. Goodacre, Rapid Commun. Mass Spectrom., 20: 1192-1198, 2006

[8] J.L. Edwards and R.T. Kennedy, Anal. Chem., 77: 2201-2209, 2005

[9] D. Miura, Y. Fujimura, H. Tachibana and H. Wariishi, Anal. Chem., 82: 498-504, 2010

[10] J. Wei, J.M. Buriak and G. Siuzdak, Nature, 399: 243-246, 1999

[11] S. Vaidyanathan, D. Jones, D.I. Broadhurst, J. Ellis, T. Jenkins, W.B. Dunn, A. Hayes, N. Burton, S.G. Oliver and D.B. Kell, Metabolomics, 1: 243-250, 2005

[12] S. Vaidyanathan, D. Jones, J. Ellis, T. Jenkins, C. Chong, M. Anderson, R. Goodacre, Rapid Commun. Mass Spectrom., 21: 2157-2166, 2007

[13] T.R. Northen, O. Yanes, M.T.Northen, D. Marrinucci, W. Uritboonthai, J. Apon, S.L. Golledge , A. Nordström , G. Siuzdak, Nature, 449: 1033-1036, 2007

[14] H.K. Woo, T.R. Northen, O. Yanes and G. Siuzdak, Nature Protocols, 3: 1341-1349, 2008

[15] R.H. Daniels, S. Dikler, E. Li and C. Stacey, JALA, 13: 314-321, 2008

[16] S. Colantonio, J.T. Simpson, R.J. Fisher, A. Yavlovich, J.M. Belanger, A. Puri and R. Blumenthal, Lipids, 46: 469-477, 2011 
[17] Y. Coffinier, S. Szunerits, H. Drobecq, O. Melnyk and R. Boukherroub, Nanoscale, 4: 231-238, 2012

[18] R.C. Gamez, E.T. Castellana and D.H. Russell, Langmuir, 29: 6502-6507, 2013

[19] B. Nie, B.K. Duan and P.W. Bohn, ACS Appl Mater Interfaces, 5: 6208-6215, 2013

[20] C.R. McAlpin, K.J. Voorhees, A.R. Corpuz and R.M.Richards, Anal. Chem., 84: 7677-7683, 2012

[21] C. W. Tsao and D.L. Devoe, Methods Mol, Biol.,790: 183-189, 2011

[22] A.M. Dattelbaum and S. Iyer, Expert Rev. Proteomics, 3: 153-161, 2006

[23] M. Rainer, M.N. Qureshi and G.K.Bonn, Anal. Bioanal. Chem., 400: 2281-2288, 2011

[24] P.L. Urban, A. Amantonico and R. Zenobi, Mass Spectrom Rev., 30: 435-478, 2011

[25] J.A. Kirwan, D.I. Broadhurst, R.L. Davidson, M R. Viant, Anal. Bioanal. Chem., 405: 5147-5157, 2013

[26] L. Lin, Q. Yu, X. Yan, W. Hang, J. Zheng, J. Xing and B. Huang, Analyst, 135: 2970-2978, 2010

[27] X. Han, K. Yang and R.W. Gross, Mass Spectrom. Rev., 31: 134-178, 2012

[28] R. Garrett, B.G. Vaz, A.M. Hovell, M.N. Eberlin and C.M. Rezende, J. Agric. Food Chem., 60: $4253-4258,2012$

[29] S. Vichi, N. Cortés-Francisco and J. Caixach, J. Mass Spectrom., 47: 1177-1190, 2012

[30] A. Nordstrom, E. Want, T. Northen, J. Lehtio and G. Siuzdak, Anal. Chem., 80: 421-429, 2008

[31] M-Z. Huang, S-C. Cheng, Y-T. Cho and J. Shiea, Anal. Chim. Acta, 702:1-15, 2011

[32] A. Venter, M. Nefliu and R.G. Cooks, Trends in Anal Chem., 27: 284-290, 2008

[33] D.R. Ifa, A.U. Jackson, G. Paglia and R.G. Cooks, Anal. Bioanal. Chem., 394: 1995-2008, 2009

[34] D.J. Weston, Analyst, 135: 661-668, 2010

[35] Z. Takats, J.M. Wiseman, B. Gologan and R.G. Cooks, Science, 306: 471-473, 2004

[36] G.A. Harris, A.S. Galhena and F.M. Fernández, Anal. Chem., 83: 4508-4538, 2011 
[37] H. Chen, Z. Pan, N. Talaty, D. Raftery and R.G. Cooks, Rapid Commun. Mass Spectrom., 20: 15771584,2006

[38] R.G. Cooks, Z. Ouyang, Z. Takats and J.M. Wiseman, Science, 311: 1566-1570, 2006

[39] J.F. Garcia-Reyes, A.U. Jackson, A. Molina-Diaz and R.G. Cooks, Anal. Chem., 81: 820-829, 2009

[40] N.I. Joyce, C.C. Eady, P. Silcock, N.B. Perry and J.W. van Klink, J. Agric. Food Chem., 61: 14491456,2013

[41] R.B. Cody, J.A. Laramee and H.D. Durst, Anal. Chem., 77: 2297-2302, 2005

[42] J.H. Gross, Anal. Bioanal. Chem., (DOI 10.1007/s00216-013-7316-0) 2013

[43] Y. Zhao, M. Lam, D. Wu and R. Mak, Rapid Commun. Mass Spectrom., 22: 3217-3224, 2008

[44] J.Y. Yew, R.B. Cody and E.A. Kravitz, Proc Natl Acad Sci U S A, 105: 7135-7140, 2008

[45] M. Zhou, J.F. McDonald and F.M. Fernández, J. Am. Soc. Mass Spectrom., 21: 68-75, 2010

[46] C.M. Jones and F.M. Fernández, Rapid Commun. Mass Spectrom., 27: 1311-1318, 2013

[47] H. Novotná, O. Kmiecik, M. Gałązka, V. Krtková, A. Hurajová, V. Schulzová, E. Hallmann, E. Rembiałkowska and J. Hajšlová, Anal. Control Expo. Risk Assess., 29: 1335-1346, 2012

[48] K. Fraser, G.A. Lane, D.E. Otter, S.J. Harrison, S.Y. Quek, Y. Hemar and S. Rasmussen, Food Chem., 141: 2060-2065, 2013

[49] J. Hajslova, T. Cajka and L. Vaclavik, Trends Anal. Chem., 30: 204-218, 2011

[50] M. Farre, Y. Pico and D. Barcelo, Anal. Chem., 85: 2638-2644, 2013

[51] J.L. Rummel, A.M. McKenna, A.G. Marshall, J.R. Eyler and D.H. Powell, Rapid Commun. Mass Spectrom., 24: 784-790, 2010

[52] T. Cajka, K. Riddellova, P. Zomer, H. Mol and J. Hajslova, Food Addit. Contam. Part A Chem. Anal. Control Expo. Risk Assess., 28: 1372-1382, 2011

[53] E.S. Chernetsova, M. Bromirski, O. Scheibner and G.E. Morlock, Anal. Bioanal. Chem., 403: 28592867,2012 
[54] H. Wang, J. Liu, R.G. Cooks and Z. Ouyang, Angew Chem. Int. Ed. Engl., 49: 877-880, 2010

[55] Z. Zhang, W. Xu, N.E. Manicke, RG Cooks and Z. Ouyang, Anal. Chem., 84: 931-938, 2012

[56] J. Liu, H. Wang, N.E. Manicke, J.M. Lin, R.G. Cooks and Z. Ouyang, Anal. Chem., 82: 2463-2471, 2010

[57] J. Liu, H. Wang, R.G. Cooks and Z. Ouyang, Anal. Chem., 83: 7608-7613, 2011

[58] J.I. Zhang, X. Li, Z. Ouyang and R.G. Cooks, Analyst, 8: 3091-3098, 2012

[59] R. Ait-Belkacem, L. Sellami, C. Villard, E. DePauw, D. Calligaris and D. Lafitte, Trends Biotechnol., 30: 466-474, 2012

[60] D. Miura, Y. Fujimura and H. Wariishi, J. Proteomics, 75: 5052-5060, 2012

[61] A. Svatos, Trends Biotechnol., 28: 425-434, 2010

[62] T. Greer, R. Sturm and L. Li, J. Proteomics, 74: 2617-2631 , 2011

[63] P.J., Trim, M.C. Djidja, T. Muharib, L.M. Cole, B. Flinders, V.A. Carolan, S. Francese and M.R. Clench, J. Proteomics, 75: 4931-4940, 2012

[64] C. Wu, A.L. Dill, L.S. Eberlin, R.G. Cooks and D.R. Ifa, Mass Spectrom. Rev., 32: 218-243, 2013

[65] R.J. Goodwin, J. Proteomics, 75: 4893-4911, 2012

[66] J.D. Watrous and P.C. Dorrestein, Nat Rev Microbiol. 9: 683-694, 2011

[67] R.M. Caprioli, T.B. Farmer and J. Gile, Anal. Chem., 69: 4751-4760, 1997

[68] S. Kaspar, M. Peukert, A. Svatos, A. Matros and H-P. Mock, Proteomics, 11: 1810-1850, 2011

[69] N. Zaima, N. Goto-Inoue, T. Hayasaka and M. Setou, Rapid Commun. Mass Spectrom., 24: 27232729,2010

[70] N. Zaima, N. Goto-Inoue, T. Hayasaka, H. Enomoto and M. Setou, Anal. Bioanal. Chem., 400: 1865-1871, 2011

[71] F. Benabdellah, D. Touboul, A. Brunelle and O. Laprevote, Anal. Chem., 81: 5557-5560, 2009 
[72] S. Khatib-Shahidi, M. Andersson, J.L. Herman, T.A. Gillespie and R.M. Caprioli, Anal. Chem., 78: 6448-6456, 2006

[73] D. Miura, Y. Fujimura, M. Yamato, F. Hyodo, H. Utsumi, H. Tachibana and H. Wariishi, Anal. Chem., 82: 9789-9796, 2010

[74] M.M. Burrell, C.J. Earnshaw and M.R. Clench, J. Exp. Bot., 58: 757-763, 2007

[75] R. Calavia, F.E. Annanouch, X. Correig and O. Yanes, J. Proteomics, 75: 5061-5068, 2012

[76] M.P. Greving, G.J. Patti and G. Siuzdak, Anal. Chem., 83: 2-7, 2011

[77] G.J. Patti, L.P. Shriver, C.A. Wassif, H.K. Woo, W. Uritboonthai, J. Apon, M. Manchester, F.D. Porter and G. Siuzdak, Neuroscience, 170: 858-864, 2010

[78] R.M. Sturm, T. Greer, R. Chen, B. Hensen and L. Li, Anal Methods, 5: 1623-1628, 2013

[79] A. Tata, A.M. Fernandes, V.G. Santos, R.M. Alberici, D. Araldi, C.A. Parada, W. Braguini L. Veronez, G. Silva Bisson, F.H. Reis, L.C. Alberici and M.N. Eberlin, Anal. Chem., 84: 6341-6345, 2012

[80] Y.J. Lee , D.C.Perdian , Z.H. Song, E.S. Yeung and B.J. Nikolau, Plant J., 70: 81-95, 2012

[81] A. Zavalin, J. Yang and R. Caprioli, J. Am. Soc. Mass Spectrom., 24: 1153-1156, 2013

[82] R. Castaing and G.J. Slodzian, Microscopie, 1: 395-399, 1962

[83] H.A. Klitzing, P.K. Weber and M.L Kraft, Methods Mol. Biol., 950: 483-501, 2013

[84] J.S. Fletcher, N.P. Lockyer and J.C. Vickerman, Surf. Interface Anal,. 43: 253-256, 2011

[85] M.K. Passarelli and N. Winograd, Biochim Biophys Acta., 1811: 976-990, 2011

[86] E.J. Lanni, S.S. Rubakhin and J.V. Sweedler, J. Proteomics, 75: 5036-5051, 2012

[87] D.F. Smith, A. Kiss, F.E. Leach, E.W. Robinson, L. Paša-Tolić and R.M.A. Heeren, Anal. Bioanal. Chem., 405: 6069-6076, 2013

[88] J.M. Wiseman, D.R. Ifa, Q. Song and R.G. Cooks, Angew. Chem. Int., 45: 7188-7192, 2006

[89] D.R. Ifa, J.M. Wiseman, Q. Song and R.G. Cooks, Int. J. Mass Spectrom., 259: 8-15, 2007

[90] J. Thunig, S.H. Hansen and C. Janfelt, Anal. Chem. 83: 3256-3259, 2011 
[91] A.L. Dill, D.R. Ifa, N.E. Manicke, Z. Ouyang and R.G. Cooks, J. Chromatogr. B, 877: 2883-2889, 2009

[92] B. Li, N. Bjarnholt, S.H. Hansen and C. Janfelt, J. Mass Spectrom., 46: 1241-1246, 2011

[93] R.G. Hemalatha and T. Pradeep, J. Agric. Food Chem., 61: 7477-7487, 2013

[94] P. Nemes, A.A. Barton and A. Vertes, Anal. Chem., 81: 6668-6675, 2009

[95] P. Nemes, A.S. Woods, A. Vertes, Anal. Chem., 82: 982-988, 2010

[96] P. Nemes and A. Vertes, Anal. Chem., 21: 8098-8106, 2007 


\section{FIGURES}

Figure 1. Schematic illustration of the inter-relationship among the MS/MS techniques for the analysis of individual molecular species of a class of interest. We only illustrate the analysis of three species (M1, M2, and M3) of a class for simplicity, whereas there exist up to hundreds of individual molecular species within a class. We assume that this class of lipid species, similar to a class of phospholipids or sphingolipids possesses one common neutral-loss fragment with mass of $\mathrm{m}_{\mathrm{a}}\left(\mathrm{i} \cdot \mathrm{e} ., \mathrm{M}_{1 \mathrm{a}}=\mathrm{M} 2-\mathrm{m}_{2 \mathrm{a}}=\right.$ $\mathrm{M} 3-\mathrm{m}_{3 \mathrm{a}}=\mathrm{m}_{\mathrm{a}}\left(\mathrm{a}\right.$ constant)), one common fragment ion at $\mathrm{m} / \mathrm{z} \mathrm{m}_{\mathrm{c}}$ (i.e., $\mathrm{m}_{1 \mathrm{c}}=\mathrm{m}_{2 \mathrm{c}}=\mathrm{m}_{3 \mathrm{c}}=\mathrm{m}_{\mathrm{c}}$ ), and a specific ion to individual species at $\mathrm{m} / \mathrm{z} \mathrm{m}_{1 \mathrm{~b}}, \mathrm{~m}_{2 \mathrm{~b}}$, and $\mathrm{m}_{3 \mathrm{~b}}$, respectively, which might not be identical to each other. Specifically, the common neutral fragment and the common fragment ion both result from the head group of the class; the individual species-specific ions represent the fatty acyl moieties of the species; and thus the residual part of each individual species can be derived from these fragments in combination with the $\mathrm{m} / \mathrm{z}$ of each molecule ion. Panel A shows a simplified full-mass scan; Panel B illustrates the product-ion analysis of these molecule ions; Panel $\mathrm{C}$ demonstrates the scanning of the individual neutral-loss fragment between a specific molecule ion and its individual fragment ion; and Panel D represents the scanning of each individual fragment ion. It should be emphasized that, although the analyses of fragments with either neutral-loss scanning (NLS) or precursor-ion scanning (PIS) are much more complicated than those in product-ion scanning in this simplified case, the analyses by NLS or PIS are much simpler than that with product-ion scanning. (Reproduced from [27]).

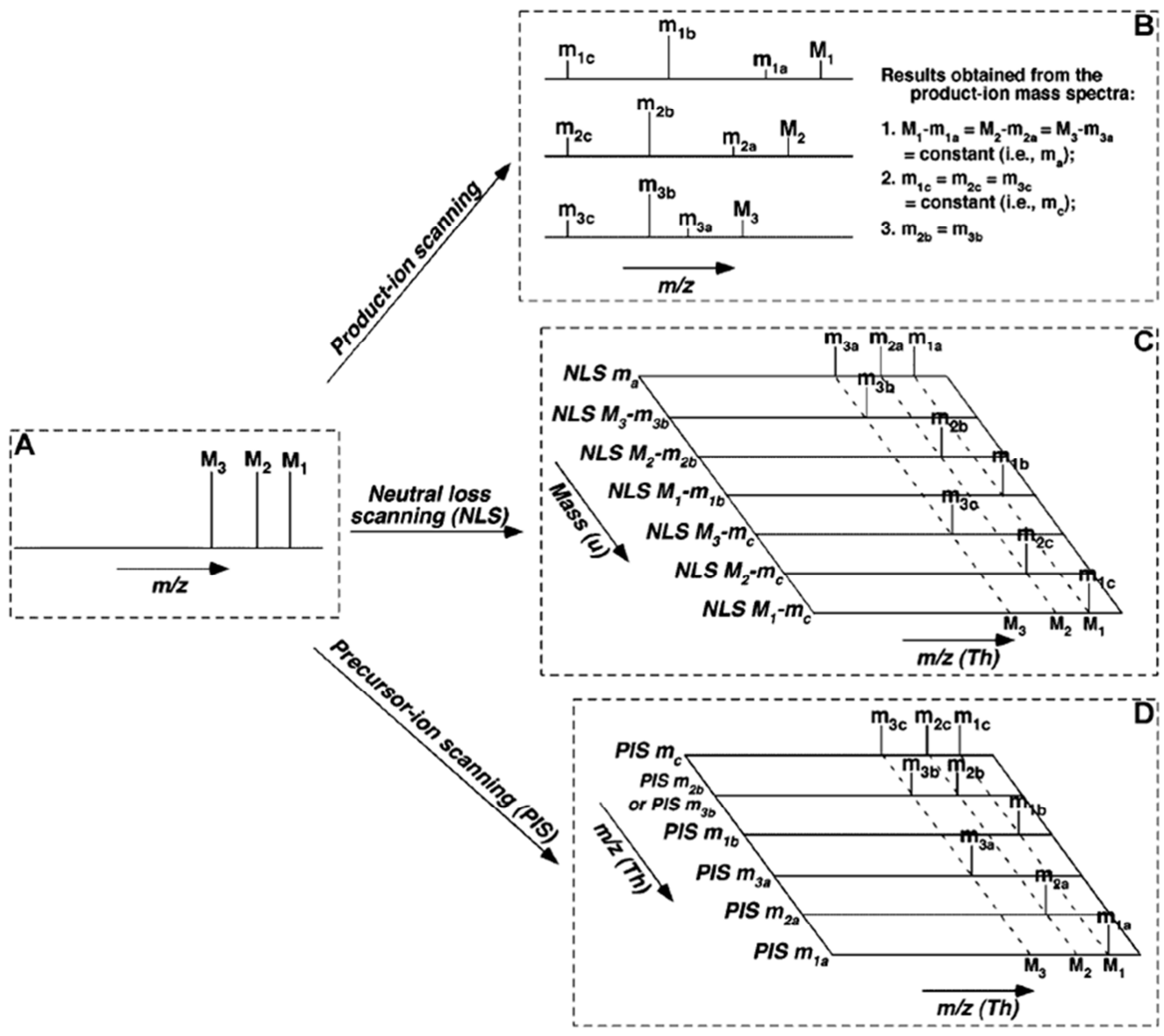


Figure 2. Schematic showing the DESI analyses for ambient high-throughput MS of unprepared samples. (Reproduced from [38]).

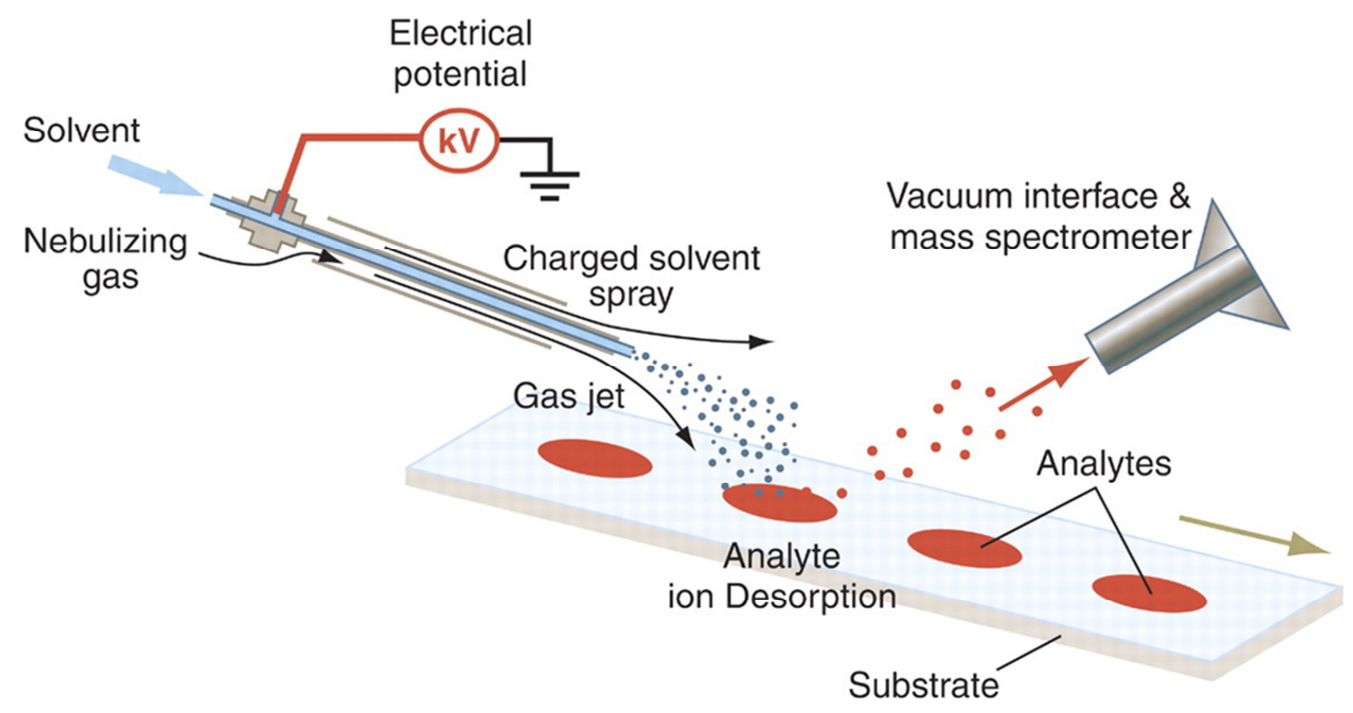


Figure 3. Schematic showing the DART analyses for ambient high-throughput MS of unprepared samples. (Reproduced from [38]).

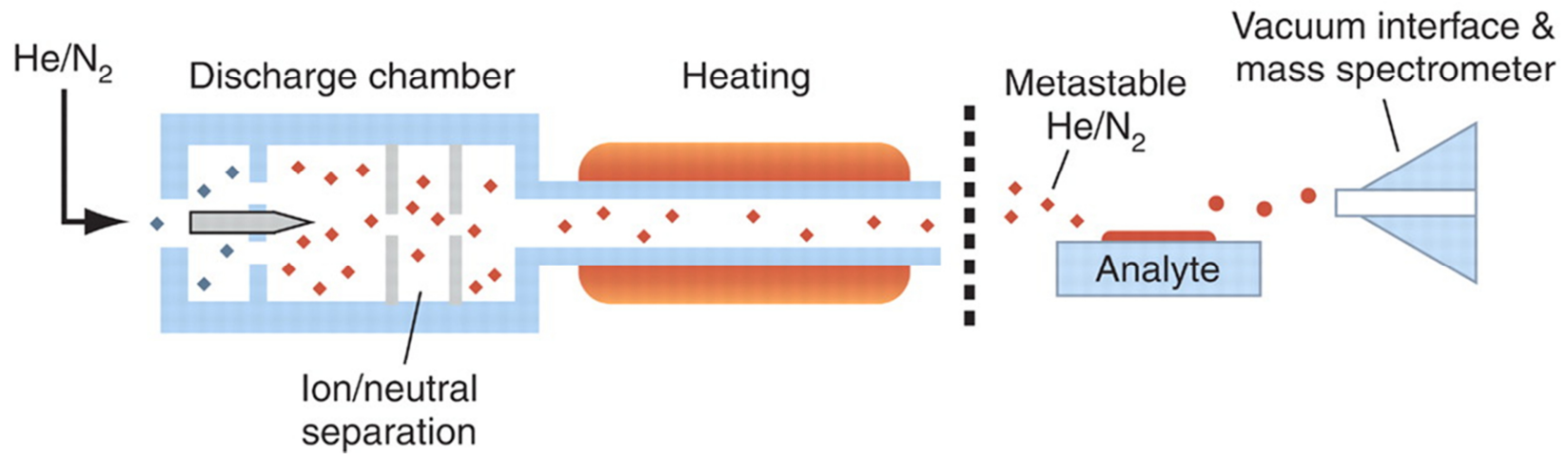


Figure 4. (a) Photograph of leaf spray ionization of green onion leaf cut to a point and held by a high voltage connector in front of the atmospheric inlet of a mass spectrometer. (b) Leaf spray spectrum acquired from green onion leaf in positive ion mode, showing sucrose and glucose ions. (c) Photograph of leaf spray ionization of spinach leaf in negative ion mode. The spinach leaf was cut into a triangle, and methanol was applied on the leaf to achieve leaf spray ionization. (d) Leaf spray spectrum acquired from spinach leaf, showing amino acids and organic acids. (e) Leaf spray spectrum acquired from peanut seed in negative ion mode, showing three fatty acids. (f) Leaf spray spectrum acquired from cranberry fruit in positive ion mode, showing a series of phytochemicals. Assignments given are based on exact mass and/or MS/MS data. (Reproduced from [57]).
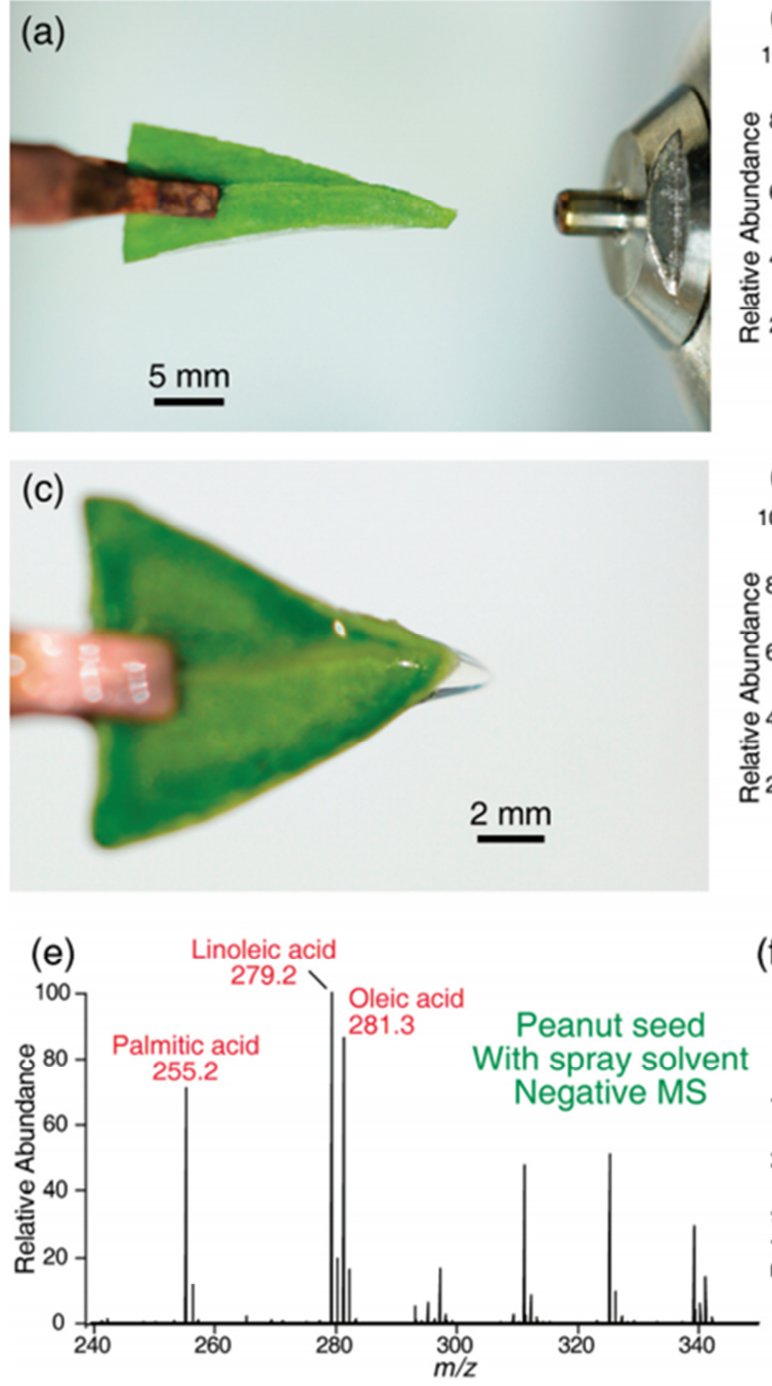
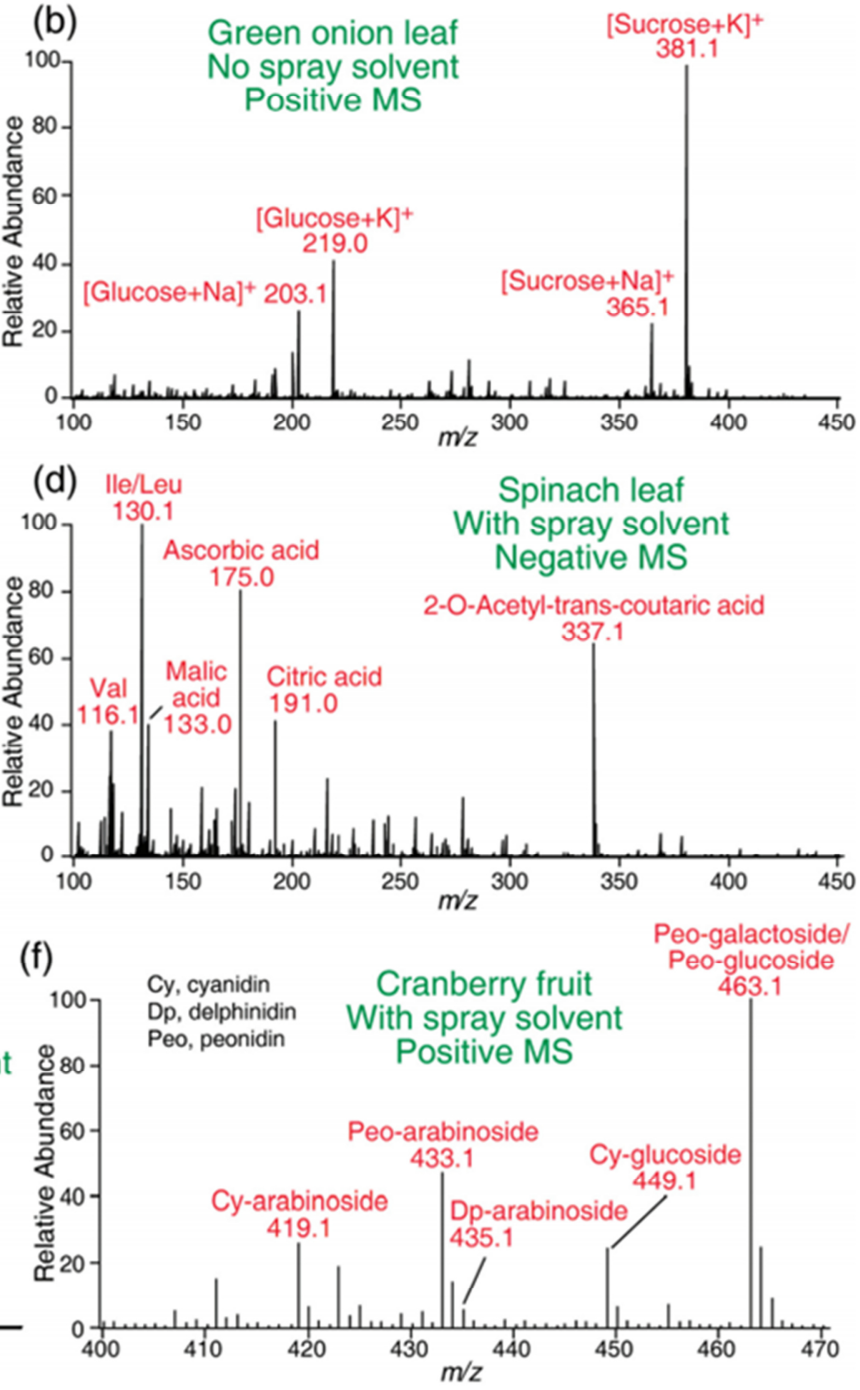
Figure 5. MSI imaging concepts and methods. (a) In a typical MSI experiment the total area is subdivided (conceptually) into pixels that are individually inspected. (b) For each pixel a single mass spectrum or the average of several mass spectra is collected and stored together with its spatial coordinates. (c) After the entire surface is scanned, an average mass spectrum can be created. The distribution of specific ions can be visualized by the creation of chemical images where the color scale (false color) represents the normalized intensity of particular ions. Each pixel from the image is associated with the original mass spectrum/mass spectra acquired at the specific point. The numbers 1 and 2 on panel a, represent the steps desorption and ionization process. (d) The aim of imaging is to display the distribution of chemicals across a surface. (Reproduced from [64]).

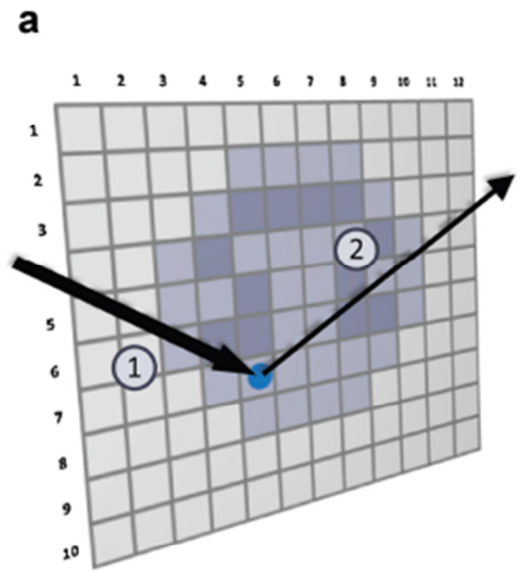

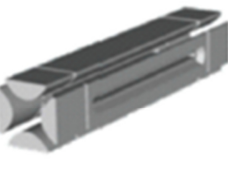

MS analysis, detection and data storage.
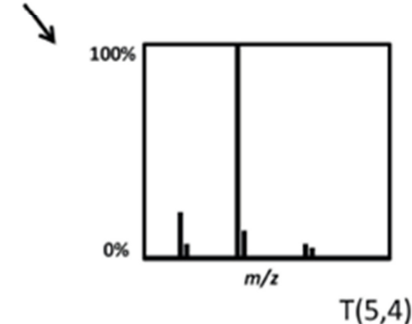

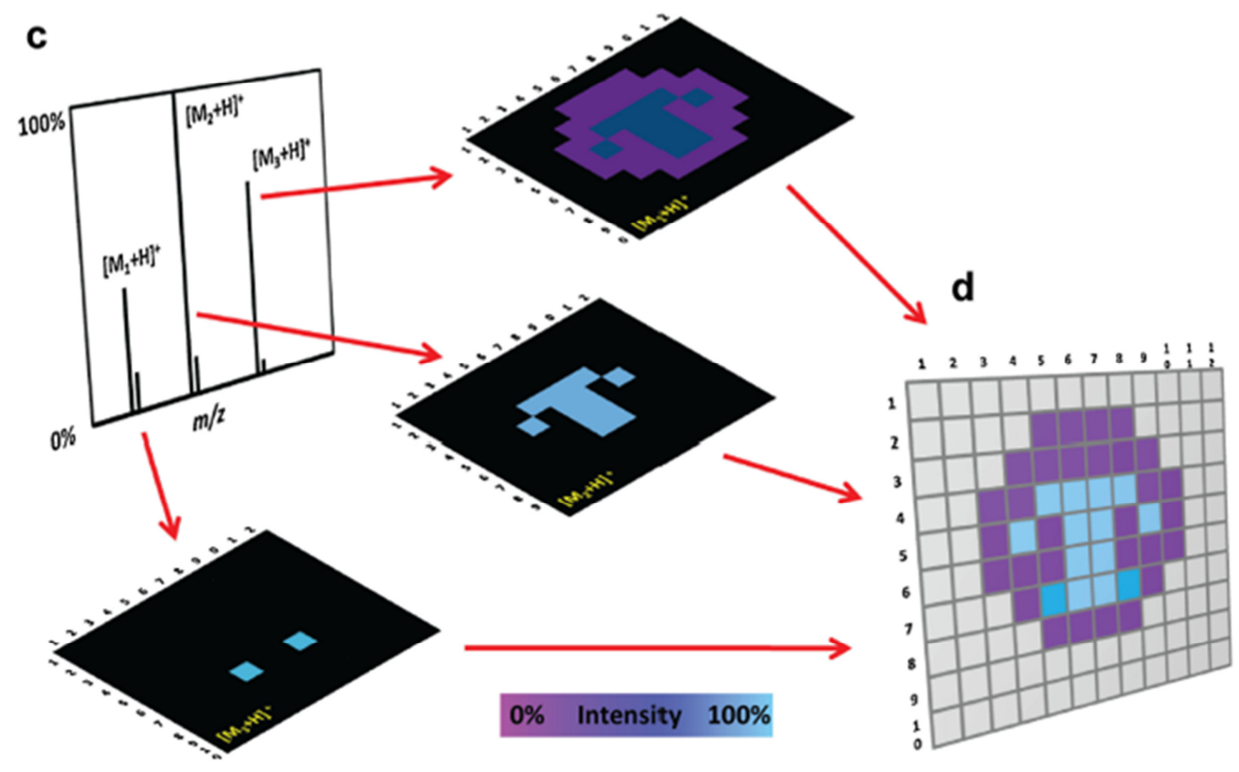


Figure 6. MALDI chemical images of (a) AMP, (b) ADP, (c) UDP-GlcNac, (d) F-1,6-biP, and (e) GTP acquired in the negative ion mode from a rat brain section deposited on a stainless steel plate, after deposition of a homogeneous layer of 9-AA. Field of view: $8.3 \times 8.3 \mathrm{~mm} 2$, pixel size $50 \mu \mathrm{m}$. The values of intensity (I) indicated under each image correspond to the minimal and maximal intensity in a pixel. (f) Optical image of a brain tissue section after 9-AA deposition and analysis by MALDI imaging with a $50 \mu \mathrm{m}$ pixel size. (Reproduced from [71]).
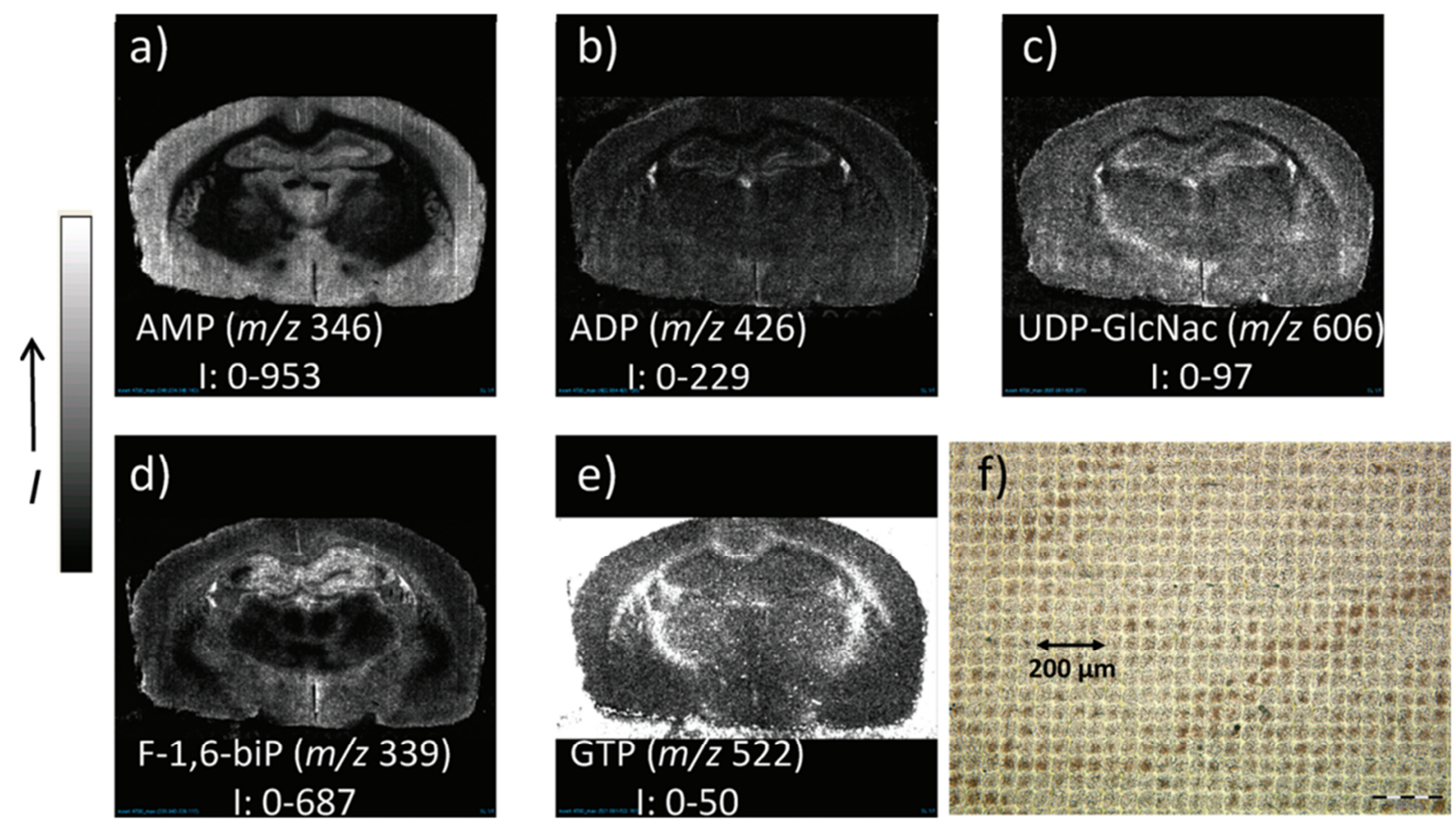
Figure 7. Schematic showing the SIMS analyses for imaging mass spectrometry. (Reproduced from http://www.geobiologie.unigoettingen.de/people/vthiel/tof_sims/index_e.shtml)

\section{Pulsed primary ion beam}

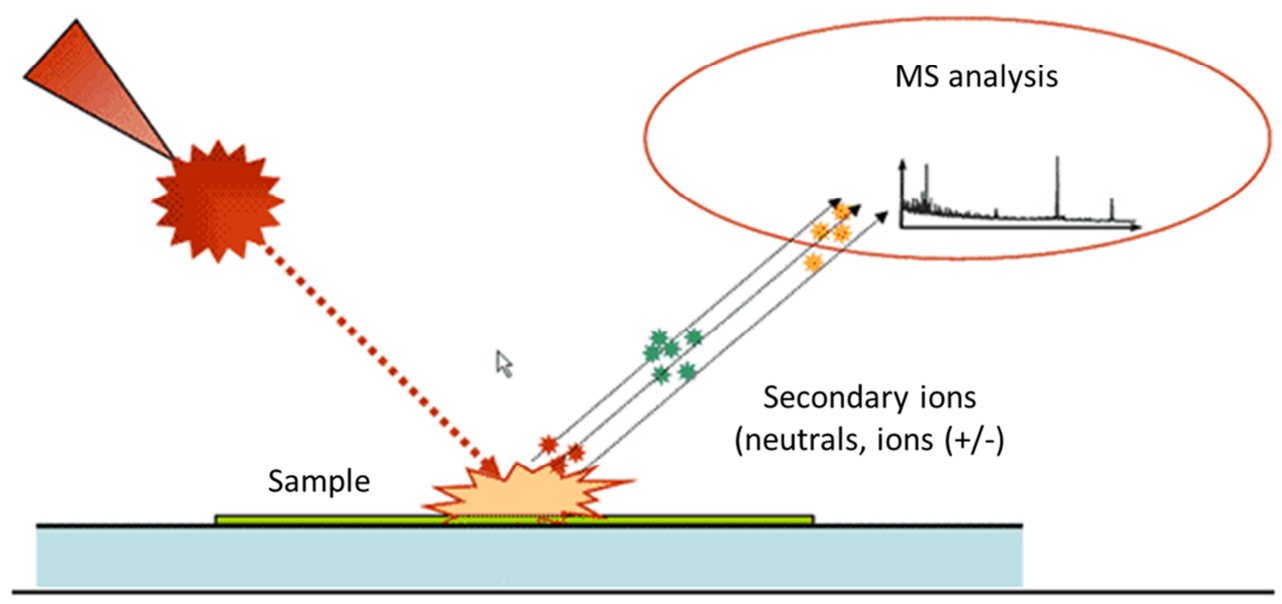


Figure 8. Photographs of flowers, petals, and a leaf of Madagascar periwinkle C. roseus and their TLC imprints: Images of (A) pink flower, (a1) single petal of a pink flower, and (a2 ) TLC-imprint of a pink petal. Images B, b1, and b2 correspond to the same data for a white flower. Images $\mathrm{C}$ and $\mathrm{c} 1$ correspond to a leaf and its imprint. Imprints do not correspond to the same petals or leaf whose photographs are shown. Images D and E correspond to one of the DESI MS images collected from petal and leaf showing the difference in spatial distribution between purple and white varieties of periwinkle, using the ion at $\mathrm{m} / \mathrm{z} 337 \mathrm{and} 457$, respectively. Scale bars of both the images in D and $\mathrm{E}$ are the same $(5 \mathrm{~mm})$. (Reproduced from [93]).
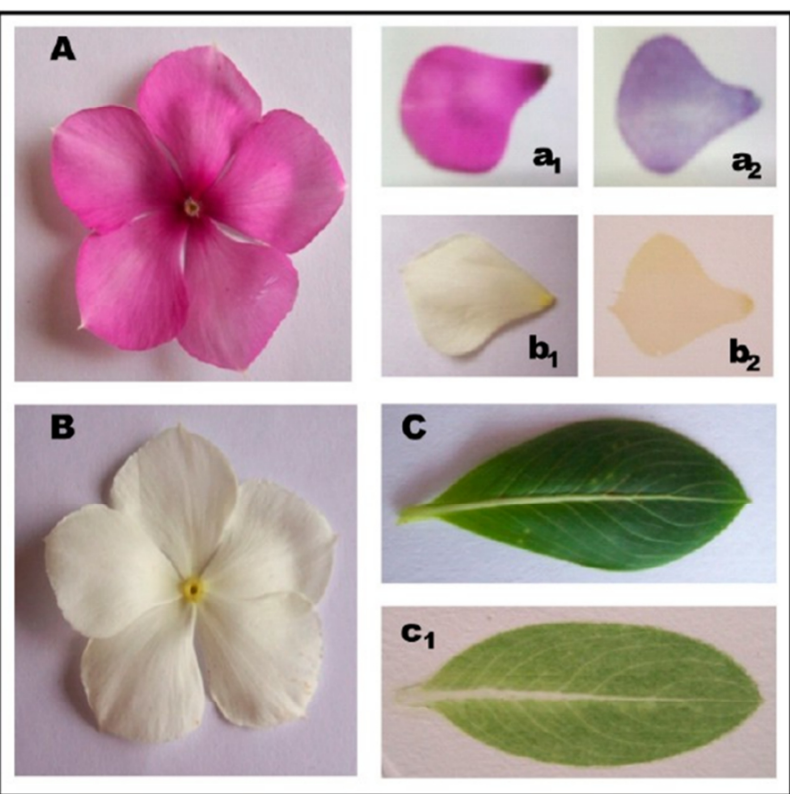



E Leaf of purple variety Leaf of white variety
